\title{
GEOMETRIC STRUCTURE OF MULTIPLE TIME-SCALE NONLINEAR SYSTEMS
}

\author{
Sanjay Bharadwaj * Kenneth D. Mease* \\ * Department of Mechanical and Aerospace Engineering \\ University of California, Irvine \\ Irvine, CA-92697, U.S.A \\ Email : sanjay@eng.uci.edu,kmease@uci.edu
}

\begin{abstract}
The geometric structure of finite-dimensional nonlinear systems with dynamics on multiple time-scales is studied by analyzing the time-scale structure present in the associated linear variational system which evolves on the tangent bundle to the state-space. As a first step, we restrict our attention to a chosen reference orbit of the nonlinear system and analyze the time scales of the linear time-varying (LTV) system that arises from linearization about that reference trajectory. We use Lyapunov exponents and associated characteristic directions to characterize the time-scales in the LTV system and their geometry. This information is used to construct decoupling coordinate transformations. An example elucidates the methodology described.
\end{abstract}

Keywords: Decomposition Methods; Decoupling; Transformations; Differential Geometric Methods

\section{INTRODUCTION}

Scientists and engineers of today are required to analyze, design, simulate and control systems of ever increasing complexity. These systems usually display dynamical behavior on widely separated time-scales and their mathematical models are usually nonlinear and high dimensional. The potential advantages afforded by knowledge of the time-scale structure present in a system provides strong motivation for this research. For example, it affords the opportunity for simplified analysis by decomposing the original model into subsystems with distinct time-scale and/or stability properties. Also, simulations of complex nonlinear systems with multiple time-scales can benefit from numerical algorithms that are able to account for and exploit the time-scale separation in order to ensure accuracy.

Interesting insight on constructing general methods to effect this desired time-scale decomposition of the mathematical model of the original system into reduced order subsystems can be obtained by studying the geometric structure of the flow of the dynamical system in the underlying state space. However, unlike linear time-invariant (LTI) systems of the general form $\dot{x}=A x$, where $x \in$ $\mathbb{R}^{n}$ and $A \in \mathbb{R}^{n \times n}$ whose time-scale structure and geometry are completely characterized by the eigenstructure of the matrix $A$, no such general tools are known for nonlinear systems. In some restricted cases, such as nonlinear systems that are in the standard form of a singularly perturbed system (Kokotovic, 1986), some tools exist for time-scale analysis. However, casting a general problem in standard from requires a priori knowledge of variables that correspond to the different time-scales present. Physical insight can be useful for low-dimensional problems in arriving at the standard form but as system dimension and complexity increase, physical insight diminishes. No systematic and tractable method is known to transform a generic nonlinear system into standard form. In this work, we attempt to investigate the time-scale structure of generic smooth finite- 
dimensional nonlinear dynamical systems by using a differential geometric viewpoint without imposing a priori restrictions on their form.

\section{APPROACH}

To analyze the time-scale structure of systems whose dynamics are represented by nonlinear differential equations of the form

$$
\dot{x}=f(x), x \in \mathbb{R}^{n}
$$

we investigate the flow on the tangent bundle $T \mathbb{R}^{n}$ specified by the vector field $f(\cdot)$ together with its associated linear variational system of equations

$$
\delta \dot{x}=(\partial f / \partial x) \delta x=A(x(t)) \delta x, \delta x \in T_{x} \mathbb{R}^{n}
$$

Let $\phi_{t}(\cdot)$ denote the flow of the nonlinear dynamics (1). Let $D \phi_{t}(x)(\cdot)$ denote the flow of the variational system $(2)$. We seek to decompose the tangent space $T_{x} \mathbb{R}^{n}$ at each $x \in N \subset \mathbb{R}^{n}$, in some region of the state space given by an open set $N \subset$ $\mathbb{R}^{n}$, into subspaces with distinct time-scale and/or stability properties. We restrict our attention to a bounded region $N$ of the state-space over which the time-scale separation is assumed to be uniform because nonlinear dynamics are capable of exhibiting different time-scale separation characteristics in different regions of the state-space. Hence, we seek a set of linearly independent basis vectors $e_{i}(x), i=1, \ldots, n$ that varies smoothly with $x$ on each tangent space $T_{x} N$ (analogous to the eigenvectors of an LTI system) that identify time-scale splitting directions. We require these directions to define locally invariant distributions (Isidori, 1994). For example, suppose each tangent space can be decomposed into a $p$-dimensional "slow" subspace spanned by vectors $\left\{e_{1}, \ldots, e_{p}\right\}$ and an $(n-p)$-dimensional "fast" subspace spanned by vectors $\left\{e_{p+1}, \ldots, e_{n}\right\}$ then the slow distribution is given by $\Delta^{s}(x)=\operatorname{span}\left\{e_{1}(x), \ldots, e_{p}(x)\right\}$ and the fast distribution is given by $\Delta^{f}(x)=$ $\operatorname{span}\left\{e_{p+1}(x), \ldots, e_{n}(x)\right\}$ where each $e_{i}(x) \in$ $T_{x} N$ and is a smooth function of $x$. Local invariance implies that for any $v \in \Delta^{s}\left(x_{0}\right) \subset T_{x_{0}} N$ where $x_{0} \in N \subset \mathbb{R}^{n}, D \phi_{t}\left(x_{0}\right)(v) \in \Delta^{s}(x) \subset T_{x} N$ for all $x \in\left\{x: x=\phi_{t}\left(x_{0}\right)\right\}$ where the time $t$ is restricted up to when the flow $\phi_{t}\left(x_{0}\right)$ first departs from the neighborhood $N$. This should hold for arbitrary $x_{0} \in N$. In fact, such a decomposition corresponds to splitting the tangent bundle $T N=\cup_{x \in N} T_{x} N$ into invariant subbundles with distinct time-scale and/or stability properties; in the above example $T_{x} N=\Delta^{s}(x) \oplus \Delta^{f}(x)$ for all $x \in N$. The Frobenius theorem (Isidori, 1994) states that if for example the slow distribution $\Delta^{s}(x)$ is involutive, then it defines a family of submanifolds of the base manifold $\mathbb{R}^{n}$. One of this foliation of submanifolds is the slow submanifold. Additionally, if the directions satisfy the more stringent condition $\left[e_{i}(x), e_{j}(x)\right]=0$ for any $1 \leq$ $i, j \leq n$, and for all $x \in N$ where $[\cdot, \cdot]$ denotes the Lie-bracket, then these directions can be used to define new local coordinates which decompose the nonlinear system into fast and slow subsystems.

As a step toward this end, we first analyze the time-scale structure of the nonlinear system (1) in the vicinity of a reference orbit $\gamma(\cdot) \in N$ by studying the linear time-varying (LTV) system of differential equations

$$
\delta \dot{x}=A(\gamma(t)) \delta x=A(t) \delta x
$$

obtained from the variational equations. The LTV system determines how tangent vectors are mapped between the different tangent spaces $T_{\gamma(t)} N$ along the reference trajectory. Knowledge of tangent vectors that grow/decay at extremal rates when mapped by the flow determined by the LTV system and their corresponding rates of expansion/contraction yields information about convergence/divergence of state trajectories neighboring $\gamma(\cdot)$ and their corresponding rates. Let $U$ denote the set of points in the statespace orbit $\gamma(\cdot)$ so that $T U=\bigcup_{\gamma(t)} T_{\gamma(t)} N$ denotes the restriction of the tangent bundle $T N$ to the reference trajectory. A means of decomposing the tangent bundle $T U$ into invariant subbundles which have extremal time-scale behavior is to determine linearly independent time-varying basis vectors that separate the time-scales present in the LTV system and are invariant under the linear flow. In order to facilitate rigorous mathematical treatment of growth/decay rates of solutions, we shall assume that the reference trajectory and the LTV system (3) are well defined for all times $t \in \mathbb{R}$. Recall that the flow of the LTV system $D \phi_{t}(\gamma(\tau))(\delta x(\tau))$ factors as $D \phi_{t}(\gamma(\tau))(\delta x(\tau))=$ $\Phi(t, \tau) \delta x(\tau)$. Here $\Phi(t, \tau) \in \mathbb{R}^{n \times n}$ is the state transition matrix determined by

$$
\frac{\partial \Phi}{\partial t}=A(t) \Phi, \Phi(\tau, \tau)=I_{n}
$$

where $I_{n}$ is the identity matrix of order $n$. In this notation $\delta x(t)=\Phi(t, \tau) \delta x(\tau)$ for all $t, \tau \in \mathbb{R}$. The reader is referred to (Brockett, 1970) for a detailed description of different properties of the transition matrix. Associated with the LTV system (3) is the adjoint LTV system given by

$$
\delta \dot{\hat{x}}=-A^{T}(t) \delta \hat{x}
$$

Let the transition matrix of the adjoint LTV system (5) be $\Psi(t, \tau)$. Recall that $\Phi^{T}(t, \tau)=\Psi(\tau, t)$. $\Psi(\tau, t)$ is the transition matrix for backward time propagation of solutions from $t$ to $\tau$ of the adjoint LTV system.

\subsection{Related Previous Work}

The dynamics on the tangent bundle $T U$ are given by the LTV system (3). If the reference 
trajectory is an equilibrium point of original nonlinear system, then the resulting system is LTI. The time-scale structure of LTI systems and their geometry is very well understood (Hale, 1969). When the reference trajectory is a closed periodic orbit of the nonlinear system, then the resulting LTV system in periodic and the time-scales and their geometry are described by Floquet theory (Hale, 1969). However, an accurate characterization of time-scale structure in generic LTV systems obtained when $\gamma(\cdot)$ is not necessarily either an equilibrium nor a periodic trajectory, has remained elusive. This problem has received considerable attention starting from the pioneering work of Lyapunov (reprinted (1992)). See (Hahn, 1967) for a description of fundamental theorems relating to the spectral theory of general LTV systems. In recent times, contributions toward a spectral theory of general LTV systems can be found in the work of $\mathrm{Wu}$ (1980) and Zhu (1995). Zhu (1995) has surveyed the progress and challenges in the development of a spectral theory for general LTV systems.

Wu (1980) introduced a notion of time-varying "eigenvalues" and "eigenvectors" which would serve as more general counterparts of the eigenvalues and eigenvectors of an LTI system. However, Wu (1980) recognized that this definition does not uniquely determine the "eigenvalue""eigenvector" pairs. In fact, it is known (Hahn, 1967; Wiesel, 1994) that any set of linearly independent solutions of Eq.(3) can be used to construct a set of "eigenvectors" and corresponding "eigenvalues". Wiesel (1994) recognized the need to impose additional constraints to uniquely identify extremal growth/decay rates and corresponding directions and has used finite time counterparts of Lyapunov exponents and directions for that purpose.

2.1.1. Lyapunov Exponents Lyapunov exponents were first introduced by Lyapunov (reprinted (1992)) to study the average exponential rate of growth/decay of functions. Let $g(t)$ be real valued function defined for $t>0$ and bounded for finite $t$. Then the long time exponential behavior of $g(t)$ is captured by its Lyapunov exponent defined as

$$
\mu_{i}[g]=\lim \sup _{t \rightarrow \infty} \frac{1}{t} \log (|g(t)|)
$$

This measure of exponential growth/decay rate can be applied to the 2-norm of solutions to an LTV system given by Eq.(3). There exits a special set of linearly independent basis solutions of the LTV system, termed a normal basis which is characterized by the property that the Lyapunov exponent of any solution of the LTV system is one among the exponents of the normal basis. See (Hahn, 1967) for a detailed description of
Lyapunov exponents (also termed 'order numbers') of ordinary differential equations. For regular LTV systems, the Lyapunov exponents are given by (Dieci, 1997)

$$
\mu_{i}\left[\delta x_{i}\right]=\lim _{t \rightarrow \infty}\left(\frac{1}{t-\tau}\right) \ln \left(\left\|\Phi(t, \tau) \delta x_{i}(\tau)\right\|\right)
$$

where $\delta x_{i}(t), 1 \leq i \leq n$ constitute a set of normal basis solutions.

It is important to note that the Lyapunov exponents are constants over the entire trajectory $\gamma(\cdot)$ and are independent of the starting point $\gamma(\tau)$. Also, the exponents are known to be invariant under Lyapunov transformations (Hahn, 1967). Lyapunov exponents computed for an LTI system yield the real parts of the eigenvalues and when computed for a periodic LTV system yield the real parts of the Floquet exponents (see (Hahn, 1967),Thm. 63.4). Lyapunov exponents can therefore be considered good measures of the time-scales present in a general LTV system. It is not surprising that they have received a lot of attention both from theoretical and computational standpoints. However, the Lyapunov characteristic directions have not received as much attention.

2.1.2. Lyapunov Characteristic Directions The Lyapunov characteristic directions also called the Lyapunov vectors at any given time along the reference trajectory $\gamma(\cdot)$ point in the direction of extremal average rates of growth/decay. So, they can be determined at any initial time $\tau$ by vectors $\delta x_{i}(\tau)$ which extremize $\left\|\Phi(t, \tau) \delta x_{i}(\tau)\right\|$. The solutions $\delta x_{i}(\tau)$ to this extremization problem is given by the eigenvectors of $\lim _{t \rightarrow \infty}\left(\Phi^{T}(t, \tau) \Phi(t, \tau)\right)^{1 / 2 t}$ (Dieci et. al., 1997).

Greene and Kim (1987) have shown that when the time-scales given by the Lyapunov exponents are distinct, the corresponding Lyapunov directions depend only on the position in state space given by $\gamma(\tau)$. The Lyapunov direction fields are everywhere orthogonal and determine directions at each point on the reference trajectory along which the average growth/decay rate is extremal.

However, not all Lyapunov direction fields are invariant when propagated using the transition matrix. We have shown (Bharadwaj, 1999) that the Lyapunov vector field corresponding to the smallest Lyapunov exponent alone is invariant under the linear flow and reduces to the eigenvector corresponding to the smallest eigenvalue in the LTI case (where the eigenvalues are all real and distinct) and the Floquet direction field corresponding to the smallest Floquet exponent in the periodic LTV case (where the Floquet exponents are all real and distinct). The Lyapunov vector fields are in fact the time-varying analogs of the Schur vectors of an LTI system. This paves 
the way to construct theoretical tools for spectral analysis and decomposition of general LTV systems that subsumes the well known methods for LTI and periodic LTV systems. We now state (see (Bharadwaj and Mease, 1999) for proof) an important result that is necessary to construct decoupling coordinate transformations.

Theorem 1. The Lyapunov direction fields corresponding to the $k$ smallest Lyapunov exponents define an invariant distribution $\Delta(\gamma(t))=$ $\operatorname{span}\left\{l_{n-k+1}(t), \ldots, l_{n}(t)\right\}$.

Invariance implies that if a vector $v \in \Delta(\gamma(\tau))$ at some time $\tau$, then when propagated forward to time $t$ by the linear flow $\Phi(t, \tau) v \in \Delta(\gamma(t))$ for all $t>\tau$.

To construct decoupling coordinate transformations we need to determine analogous quantities for the associated adjoint LTV system (5) in backward time. Note that the adjoint LTV system in backward time has the same Lyapunov exponents as the original LTV system. Let the Lyapunov vector fields of the adjoint LTV system in backward time (which we shall term adjoint Lyapunov direction fields) be denoted $\hat{l}_{i}^{T}(t)$ where $\hat{l}_{i}(t)$ is a row vector. An invariance result analogous to Theorem 1 can be shown for the distribution spanned by the adjoint Lyapunov direction fields corresponding to the same $k$-smallest Lyapunov exponents. We shall now show the use the Lyapunov direction fields and the associated adjoint Lyapunov direction fields to construct decoupling coordinate transformations.

\section{DECOUPLING THE DYNAMICS ON SEPARATED TIME-SCALES}

Suppose that the Lyapunov exponents of the LTV system (3) are distinct and without loss of generality ordered as $\mu_{n}<\mu_{(n-1)}<\ldots<\mu_{1}$. Further, suppose that the LTV system has two time-scales so that the Lyapunov spectrum has a separation, i.e., $\mu_{n-k+1}<<\mu_{n-k}$. For purpose of simplicity in discussion we assume that all the exponents are negative so that the exponents $\left(\mu_{n-k+1}, \ldots, \mu_{n}\right)$ correspond to a k-dimensional "fast" subsystem $\mathcal{Q}$ and the the exponents $\left(\mu_{1}, \ldots, \mu_{n-k}\right)$ correspond to an $(n-k)$ dimensional "slow" subsystem $\mathcal{P}$. However, the method is equally applicable in the more general case when some exponents are positive.

Theorem 1 implies that distribution $\Delta^{q}$ given by the span of the Lyapunov direction fields of subsystem $\mathcal{Q}, \Delta^{q}(\gamma(\cdot))=\operatorname{span}\left\{l_{n-k+1}(\cdot), \ldots, l_{n}(\cdot)\right\}$ is invariant, i.e., the direction fields spanning $\Delta^{q}$ when propagated by the linear flow cannot develop components along $l_{1}(\cdot), \ldots, l_{k}(\cdot)$. However, note that the directions $l_{1}(\cdot), \ldots, l_{k}(\cdot)$ can under time evolution develop components along the direction fields in $\Delta^{q}$.

In order to decouple the two subsystems, we seek a Lyapunov transformation of the form $\delta x=L(t) \delta z$ that takes the dynamics (3) to a block decoupled form

$$
\begin{aligned}
\delta \dot{z} & =\left(L^{-1}(t) A(t) L(t)-L^{-1}(t) \dot{L}(t)\right) \delta z \\
& =\left[\begin{array}{cc}
A_{q}(t) & 0 \\
0 & A_{p}(t)
\end{array}\right] \delta z
\end{aligned}
$$

Note that $A_{q}(t)$ and $A_{p}(t)$ are not unique but we require $A_{q}(t)$ to have the Lyapunov exponents of subsystem $\mathcal{Q}$ and $A_{p}(t)$ to have the Lyapunov exponents of subsystem $\mathcal{P}$. However, the "fast" and "slow" distributions $\Delta^{q}$ and $\Delta^{p}$ are uniquely determined. It is known (Laub, 1991; Kokotovic et. al., 1986) that we can construct the required $L(t)$ from two simpler coordinate transformations as $L(t)=F(t) H(t)$ where

$$
\begin{aligned}
& F(t)=\left[\begin{array}{cc}
I_{k} & 0_{k \times(n-k)} \\
Q(t)_{(n-k) \times k} & I_{(n-k)}
\end{array}\right] \\
& H(t)=\left[\begin{array}{cc}
I_{k} & R(t)_{k \times(n-k)} \\
0_{(n-k) \times k} & I_{(n-k)}
\end{array}\right]
\end{aligned}
$$

The inverse transformations $F^{-1}(t)$ and $H^{-1}(t)$ can be constructed by replacing $Q(t)$ with $-Q(t)$ and $R(t)$ with $-R(t)$ respectively. Consider first the coordinate transformation $\delta x=F(t) \delta y$. With $A(t)$ suitable partitioned, the transformed dynamics are given by

$$
\begin{aligned}
\delta \dot{y} & =\left(F^{-1}(t) A(t) F(t)-F^{-1}(t) \dot{F}(t)\right) \delta y=\tilde{A}(t) \delta y \\
& =\left[\begin{array}{cc}
A_{11}(t)+A_{12}(t) Q(t) & A_{12}(t) \\
\operatorname{Ric}(Q(t), \dot{Q}(t)) & A_{22}(t)-Q(t) A_{12}(t)
\end{array}\right] \delta y
\end{aligned}
$$

If $\operatorname{Ric}(Q(t), \dot{Q}(t))=0$ so that $Q(t)$ is chosen to satisfy the differential Riccati Equation (DRE)

$$
\begin{aligned}
\dot{Q}(t)= & A_{21}(t)-Q(t) A_{11}(t) \\
& +A_{22}(t) Q(t)-Q(t) A_{12}(t) Q(t)
\end{aligned}
$$

then partial decoupling results. In order to ensure that the decoupling effected is the desired one, the solution $Q(t)$ of Eq.(11) should be so that $A_{q}(t)=A_{11}(t)+A_{12}(t) Q(t)$ and $A_{p}(t)=A_{22}(t)-$ $Q(t) A_{12}(t)$ have the Lyapunov exponents of the subsystems $\mathcal{Q}$ and $\mathcal{P}$ respectively. In these coordinates $\delta y=\left[\delta y_{q} ; \delta y_{p}\right]$, the fast variables $\delta y_{q} \in \mathbb{R}^{k}$ have no influence on the slow variables $\delta y_{p} \in$ $\mathbb{R}^{n-k}$. To complete the decoupling we employ the second coordinate transformation $\delta y=H(t) \delta z$. This results in

$$
\delta \dot{z}=\left[\begin{array}{cc}
A_{q}(t) & \operatorname{Ric}(R(t), \dot{R}(t)) \\
0 & A_{p}(t)
\end{array}\right] \delta z
$$


If $\operatorname{Ric}(R(t), \dot{R}(t))=0$ so that $R(t)$ is chosen to satisfy the DRE

$$
\dot{R}(t)=A_{q}(t) R(t)+A_{12}(t)-R(t) A_{p}(t)
$$

then the desired decoupling is effected. The composite coordinate transformation $\delta x=L(t) \delta z$ that takes the LTV system (3) to the decoupled form (8) is given by

$$
L(t)=F(t) H(t)=\left[\begin{array}{cc}
I_{k} & R(t) \\
Q(t) & Q(t) R(t)+I_{(n-k)}
\end{array}\right]
$$

Note also that

$$
\begin{aligned}
& \Delta^{q}(\gamma(\cdot))=\text { colspan }\left\{\begin{array}{c}
I_{k} \\
Q(t)
\end{array}\right\} \\
& \Delta^{p}(\gamma(\cdot))=\text { colspan }\left\{\begin{array}{c}
R(t) \\
Q(t) R(t)+I_{(n-k)}
\end{array}\right\}
\end{aligned}
$$

We shall now describe a procedure to construct the desired solution $Q(t)$ of Eq.(11) from the Lyapunov direction fields associated with subsystem $\mathcal{Q}$.

Theorem 2. Let $\Delta(\gamma(\cdot))=\operatorname{span}\left\{v_{1}(\cdot), \ldots, v_{k}(\cdot)\right\}$ be a $k$ dimensional invariant distribution. Let $V(t)_{n \times k}$ denote the matrix with columns $v_{i}(t)$. Partition $V(t)$ as $V(t)=\left[V_{11}(t)_{k \times k} ; V_{12}(t)_{(n-k) \times k}\right]$. Assuming that $V_{11}(t)$ is invertible over the desired time-interval, let $V(t) V_{11}^{-1}(t)=\left[I_{k} ; Q(t)_{(n-k) \times k}\right]$ where $Q(t)=V_{12}(t) V_{11}^{-1}(t)$. Then $Q(t)$ solves the DRE (11).

Proof : Post-multiplying $V(t)$ by the nonsingular matrix $V_{11}^{-1}(t)$ corresponds to taking linear combinations of the columns of $V(t)$ so that the resulting vectors are in the special form $\left[I_{k} ; Q(t)\right]$. Since linear combinations preserve the span and the coefficients of the linear combination form a nonsingular matrix, $\Delta(\gamma(\cdot))=\operatorname{col} \operatorname{span}\{I ; Q(\cdot)]\}$. Also, since $\Delta$ is invariant under the linear flow, the columns of $\left[I_{k}, Q(\tau)\right]$ when propagated from time $\tau$ to $t>\tau$ will lie in $\Delta(\gamma(t))$. Hence they can be expressed as some linear combinations of the columns of $\left[I_{k} ; Q(t)\right]$ which span $\Delta(\gamma(t))$ at time $t$. Therefore

$$
\Phi(t, \tau)\left[\begin{array}{c}
I_{k} \\
Q(\tau)
\end{array}\right]=\left[\begin{array}{c}
I_{k} \\
Q(t)
\end{array}\right] C(t)_{k \times k}
$$

where $C(t)$ is a nonsingular matrix of coefficients for linear combinations of the columns of $\left[I_{k} ; Q(t)\right]$. Differentiating both sides with respect to $t$ and simplifying using $\dot{\Phi}=A(t) \Phi$ and Eq.(17) we get

$$
A(t)\left[\begin{array}{c}
I_{k} \\
Q(t)
\end{array}\right] C(t)=\left[\begin{array}{c}
0 \\
\dot{Q}(t)
\end{array}\right] C(t)+\left[\begin{array}{c}
I_{k} \\
Q(\tau)
\end{array}\right] \dot{C}(t)
$$

This yields the two equations

$$
\dot{C}(t)=\left(A_{11}(t)+A_{12}(t) Q(t)\right) C(t)
$$

$$
\left(A_{21}(t)+A_{22}(t) Q(t)\right) C(t)=\dot{Q}(t) C(t)+Q(t) \dot{C}(t)
$$

Substituting for $\dot{C}(t)$ from Eq.(19) in Eq.(20) and rearranging we get

$$
\operatorname{Ric}(Q(t), \dot{Q}(t)) C(t) \equiv 0
$$

Since $C(t)$ is nonsingular, we get $\operatorname{Ric}(Q(t), \dot{Q}(t)) \equiv$ 0 , i.e., $Q(t)$ satisfies the DRE (11).

In order to effect the desired time-scale decoupling, the Lyapunov vector fields corresponding to the smallest $k$ Lyapunov exponents which span the invariant distribution $\Delta^{q}$ can be used to construct the desired solution $Q(t)$ to the DRE (11) as described above. When restricted to the case of LTI systems, the Lyapunov direction fields spanning $\Delta^{q}$ become time-invariant, i.e., Schur vectors which span an invariant subspace. The DRE (11) becomes an algebraic Riccati equation whose solution is given by a constant matrix $Q$ constructed as above using the Schur vectors.

In a similar fashion, it is possible to construct the desired solution $R(t)$ that solves Eq.(13) from the adjoint Lyapunov direction fields of the subsystem $\mathcal{Q}$.

If the LTV system (3) possesses $n$ distinct separated time-scales, they can be decoupled one at a time starting from the time-scale corresponding to the smallest Lyapunov exponent using this procedure. When the above procedure is used on a general LTV system with $n$ distinct timescales to decouple them one at a time from the rest of the time-scales, it is a generalization to LTV systems of the method of deflation used in conjunction with the power method to compute eigenvalues and eigenvectors of a constant matrix (Horn, 1985). We illustrate the decoupling procedure with an example.

Example 3. Consider the nonlinear system

$$
\begin{aligned}
& \dot{x}_{1}=-6 x_{1}+5 x_{2}+40\left(\frac{4}{9} x_{1}+\frac{5}{9} x_{2}\right)^{2} \\
& \dot{x}_{2}=4 x_{1}-5 x_{2}-32\left(\frac{4}{9} x_{1}+\frac{5}{9} x_{2}\right)^{2}
\end{aligned}
$$

This nonlinear system is designed to have slow and fast time-scales. The slow manifold of these dynamics is a parabola given by $\frac{1}{9}\left(x_{1}-x_{2}\right)=$ $\left(\frac{4}{9} x_{1}+\frac{5}{9} x_{2}\right)^{2}$. The flow on the slow manifold leads into the equilibrium point at the origin in forward time. The linearization of the nonlinear dynamics (22) about the equilibrium at the origin results in an LTI system with eigenvalues $\lambda_{1}=-1$ and $\lambda_{2}=-10$. The corresponding eigenvectors are $e_{1}=[1 ; 1]$ and $e_{2}=[5 ;-4]$.

We now linearize the nonlinear system about a reference trajectory which is contained in the slow 
manifold. The reference trajectory is given by $\gamma(t)=\left[x_{1_{\text {ref }}}(t) ; x_{2_{\text {ref }}}(t)\right]=\left[e^{-t}+5 e^{-2 t} ; e^{-t}-\right.$ $\left.4 e^{-2 t}\right]$. The resulting LTV system is given by $\delta \dot{x}=A(t) \delta x$ where

$$
A(t)=\left[\begin{array}{cc}
-6+\frac{320}{9} e^{-t} & 5+\frac{400}{9} e^{-t} \\
4-\frac{256}{9} e^{-t} & -5-\frac{320}{9} e^{-t}
\end{array}\right]
$$

By construction, the characteristic slow and fast direction fields of this LTV system are known. The slow direction at each point on the reference trajectory is given by the tangent direction $\dot{\gamma}$ since the reference trajectory is part of the slow manifold. Therefore the slow direction field $e_{1}(t)=\dot{\gamma}(t)$. So $e_{1}(t)=\left[1+10 e^{-t} ; 1-8 e^{-t}\right]$. Note that as $t \rightarrow \infty, \gamma(t)$ approaches the origin and $e_{1}(t) \rightarrow e_{1}$. By construction, the fast direction field everywhere along $\gamma(\cdot)$ is given by $e_{2}(t)=$ $[4 ;-5] . e_{1}(t)$ and $e_{2}(t)$ are the time-scale splitting direction fields and also qualify as "eigenvectors".

The transition matrix of the LTV system can also be determined in closed form and is given by $\Phi=\left[\Phi_{i j}\right]$ where

$$
\begin{aligned}
& \Phi_{11}(t)=\frac{4}{9} e^{-t}+\frac{40}{9} e^{-2 t}-\frac{35}{9} e^{-10 t} \\
& \Phi_{12}(t)=\frac{5}{9} e^{-t}+\frac{50}{9} e^{-2 t}-\frac{55}{9} e^{-10 t} \\
& \Phi_{21}(t)=\frac{4}{9} e^{-t}-\frac{32}{9} e^{-2 t}+\frac{28}{9} e^{-10 t} \\
& \Phi_{22}(t)=\frac{5}{9} e^{-t}-\frac{40}{9} e^{-2 t}+\frac{44}{9} e^{-10 t}
\end{aligned}
$$

Note that $\Phi(t, \tau)=\Phi(t, 0) \Phi^{-1}(\tau, 0)$.

It can be verified either numerically or by tedious algebra that the Lyapunov exponents of this LTV system are $\mu_{1}=-1$ and $\mu_{2}=-10$ and that the Lyapunov direction field $l_{2}(t)$ corresponding to $\mu_{2}$ points in the same direction as $e_{2}(t)$. It can also be verified either numerically or by algebraic manipulations that the adjoint Lyapunov direction field corresponding to the same time-scale, $\hat{l}_{2}(t)$, points in the direction $\left[-\left(1-8 e^{-t}\right)\left(1+10 e^{-t}\right)\right]$. Note that this direction reduces to the left-eigenvector of the LTI system corresponding to the eigenvalue $\lambda_{2}=-10$ as $t \rightarrow \infty$. Theorem 1 and its counterpart for the adjoint LTV system assure us that $\Delta^{q}=\operatorname{span}\left\{l_{2}(\cdot)\right\}$ and $\hat{\Delta}^{q}=\operatorname{span}\left\{\hat{l}_{2}^{T}(\cdot)\right\}$ are invariant distributions. Casting $l_{2}(t)$ in the form $[1 ; Q(t)]$ we get $Q(t)=-4 / 5$ which solves the DRE

$$
\begin{aligned}
\dot{Q}= & \left(4-\frac{256}{9} e^{-t}\right)-Q(t)\left(-6+\frac{320}{9} e^{-t}\right) \\
& +\left(-5-\frac{320}{9} e^{-t}\right) Q(t)-Q(t)\left(5+\frac{400}{9} e^{-t}\right) Q(t)
\end{aligned}
$$

We now construct $F(t)$ and perform the coordinate transformation $\delta x=F(t) \delta y$ so that $A_{q}(t)=$ -10 and $A_{p}(t)=-1$. We also compute $\tilde{l}_{2}(t)=$ $\hat{l}_{2}(t) F(t)=\left[\begin{array}{ll}-9 / 5 & \left(1+10 e^{-t}\right)\end{array}\right]$. Casting $\tilde{l}_{2}(t)$ in the form $[1-R(t)]$ we get $R(t)=(5 / 9)\left(1+10 e^{-t}\right)$ which solves the DRE

$$
\dot{R}=-10 R(t)+\left(5+\frac{400}{9} e^{-t}\right)+R(t)
$$

Next, we construct and perform the coordinate transformation to $\delta z$ coordinates using $H(t)$ to complete the decoupling of the slow and fast timescales. In this example the "eigenvalues" turn out to be constants -1 and -10 . Also note that the composite coordinate transformation $L(t)=$ $F(t) H(t)$ is given by

$$
L(t)=\left[\begin{array}{cc}
1 & (5 / 9)\left(1+10 e^{-t}\right) \\
-4 / 5 & (5 / 9)\left(1-8 e^{-t}\right)
\end{array}\right]
$$

Note that the columns point in the same directions as the time-scale splitting directions $e_{1}(t)$ and $e_{2}(t)$.

\section{REFERENCES}

Bharadwaj, S. and K.D. Mease (1999). A new Invariance Property of Lyapunov Characteristic Directions. Proceedings of the American Control Conference, San Diego, (Submitted).

Bittani, S., A. J. Laub and J. C. Willems (editors) (1991). The Riccati Equation. SpringerVerlag, New York.

Brockett, R. W. (1970). Finite Dimensional Linear Systems. Wiley, New York.

Dieci, L., R.D. Russell and E.S. Van Vleck (1997). On the Computation of Lyapunov Exponents for Continuous Dynamical Systems. Siam Journal of Numerical Analysis, 34, 402-423.

Greene, J.M. and J.-S. Kim (1987). The Calculation of Lyapunov Spectra. Physica D, 24, $213-225$.

Hahn, W. (1967). Stability of Motion. SpringerVerlag, New York.

Hale, J.K. (1969) Ordinary Differential Equations. Wiley-Interscience, New York.

Horn, R.A. and C.R. Johnson (1985). Matrix Analysis. Cambridge University Press, New York.

Isidori, A. (1994). Nonlinear Control Systems. Second edition, Springer-Verlag, Berlin.

Kokotovic, P.V., H. K. Khalil and J. O'Reilly (1986). Singular Perturbation Methods in Control: Analysis and Design. Academic Press, New York.

Lyapunov, A.M. (1992). The General Problem of Stability of Motion. International Journal of Control, 55, 531-773.

Wiesel, W.E. (1994). Modal Feedback on Chaotic Trajectories. Physical Review E, 49, 19901996.

Wu, M.-Y. (1980). A New Concept of Eigenvalues and Eigenvectors and its Applications. IEEE Transactions on Automatic Control, 25, 824826.

Zhu, J. (1995). A Unified Spectral Theory for Linear Time-Varying Systems - Progress and Challenges. Proceedings of the 34th IEEE Conference on Decision and Control, New Orleans, IEEE, New York, Vol. 3, pp. 25402546 . 